\title{
DIAGNÓSTICOS DE ENFERMAGEM DE PACIENTES HOSPITALIZADOS COM DOENÇAS CARDIOVASCULARES
}

\author{
Nursing diagnoses for inpatients with cardiovascular diseases \\ Diagnósticos de enfermería para pacientes hospitalizados com enfermedades \\ cardiovasculares
}

Juliana de Melo Vellozo Pereira ${ }^{1}$

Keila Mara Cassiano ${ }^{4}$
Ana Carla Dantas Cavalcanti ${ }^{2}$

Gisella de Carvalho Queluci ${ }^{5}$
Rosimere Ferreira Santana ${ }^{3}$

Tereza Cristina Felippe Guimarães ${ }^{6}$

\section{RESUMO}

Objetivo: Identificar a frequência dos diagnósticos de enfermagem e características definidoras de pacientes com doenças cardiovasculares e caracterizá-los quanto às variáveis sociodemográficas e clínicas. Método: Estudo descritivo transversal realizado com 30 pacientes hospitalizados em um hospital de grande porte. Utilizou-se instrumento próprio validado para coleta de dados, que foram analisados por 5 peritos;, havendo concordância de $50 \%$, sofreram análise estatística descritiva e inferencial. Resultados: Foram encontradas associações significativas com fatores Presença da Insuficiência Cardíaca, do Infarto Agudo do Miocárdio, da Dor, Sexo e Idade. Os diagnósticos mais frequentes foram: Ansiedade (76,7\%), Dor Aguda (70,7\%), Débito Cardíaco Diminuído (56,7\%), Percepção Sensorial Perturbada-Visual (53,3\%), Insônia (46,7\%), Intolerância à Atividade (36,7\%), Disfunção Sexual (36,7\%) e Eliminação Urinária Prejudicada (36,7\%). Conclusão: a descrição dos diagnósticos de enfermagem contribui para a análise das respostas à doença cardiovascular, com foco no objeto de trabalho do enfermeiro, apresentando respostas à doença cardiovascular por meio de investigação holística.

Palavras-chave: Diagnóstico de enfermagem. Doenças cardiovasculares. Processos de enfermagem. Enfermagem. Cardiologia.

\begin{abstract}
To identify the frequency of nursing diagnoses defining characteristics of inpatients with cardiovascular disease, and to rate them according to demographic and clinical variables. Methods: A descriptive transversal study. Data were collected through nursing assessment, medical charts, interview and physical exams with 30 inpatients in a large hospital .A proprietary instrument validated for data collection was used. Such data were analyzed by 5 experts and, upon agreement of $50 \%$, they were submitted to descriptive and inferential statistical analysis. Results: Significant associations were found with the factors Presence of Heart Failure, Acute Myocardial Infarction, Pain, Sex and Age. The most frequent nursing diagnoses were: anxiety $(76,7 \%)$, acute pain $(70,7 \%)$, decreased cardiac output $(56,7 \%)$, activity intolerance $(46,7 \%)$, sexual dysfunction $(36,7 \%)$ and impaired urinary elimination (36,7\%). The diagnosis decreased cardiac output, impaired activity and impaired urinary elimination $(p=0,029)$ were observed with high frequency in patients with heart failure $(p=0,003)$. There was also an association between acute myocardial infarction and insomnia nursing diagnosis $(p=0,014)$. Sexual dysfunction was associated with age above 60 years $(p=0,041)$. Conclusion: nursing diagnoses showed responses to cardiovascular disease through holistic research.
\end{abstract}

Keywords: Nursing Diagnosis. Cardiovascular Diseases. Nursing Process. Cardiology.

\section{Resumen}

Objetivo: Identificar la frecuencia de los diagnósticos de enfermería y las características principales de los pacientes con enfermedades cardiovasculares y darles características cuanto a las variables sociodemográficas y clínicas. Método: Estudio descriptivo transversal realizado con 30 pacientes hospitalizados en un hospital de gran porte. Se utilizó instrumento propio validado para colecta de datos, estos fueron analizados por 5 peritos y, habiendo concordancia del $50 \%$, sufrieron análisis estadística descriptiva e inferida. Resultados: fueron encontradas asociaciones significativas con los factores presencia de la insuficiencia cardíaca, del infarto agudo de miocardio, del dolor, sexo y edad. Los diagnósticos más frecuentes fueron: ansiedad $(76,7 \%)$, dolor agudo $(70,7 \%)$, disminución del gasto cardiaco $(56,7 \%)$, percepción sensorial perturbada - visual $(53,3 \%)$, insomnio $(46,7 \%)$, intolerancia a la actividad (36,7\%), disfunción sexual (36,7\%), y eliminación urinaria perjudicada (36,7\%). Conclusión: la descripción de los diagnósticos de enfermería contribuyó para el análisis de las respuestas a la enfermedad cardiovascular, enfocado en el objeto de trabajo del enfermero. Los diagnósticos de enfermería presentan respuestas a la enfermedad cardiovascular a través de investigación holística.

Palabras clave: Diagnóstico de Enfermería. Enfermedades Cardiovasculares. Procesos de Enfermería. Enfermería. Cardiología.

\footnotetext{
'Enfermeira. Mestranda em Ciências Cardiovasculares - Universidade Federal Fluminense (UFF). Niterói - RJ. Brasil. E-mail: juvellozo.uff@gmail.com.;'Doutora em Enfermagem. Professora Adjunta do Departamento de Fundamentos de Enfermagem e Administração da Escola de Enfermagem Aurora de Afonso Costa / Universidade Federal Fluminense. Niterói - RJ. Brasil. E-mail: ana_carladc@yahoo.com.br; 3Professora Adjunta do Departamento de Enfermagem Médico-Cirúrgica da Escola de Enfermagem Aurora de Afonso Costa / Federal Fluminense. Niterói - RJ. Brasil. E-mail: rosifesa@gmail.com; ${ }^{4}$ Mestre em Estatística. Professora Assistente do Instituto de Matemática e Estatística/ Universidade Federal Fluminense. Niterói - RJ. Brasil. E-mail: keilamath@hotmail.com;5Doutora em Enfermagem. Professora Adjunta do Departamento de Fundamentos de Enfermagem e Administração da Escola de Enfermagem Aurora de Afonso Costa / Universidade Federal Fluminense. Niterói - RJ. Brasil. E-mail: gisellaqueluci@yahoo.com.br; ${ }^{6}$ Mestre em Enfermagem. Enfermeira do Instituto Nacional de Cardiologia Laranjeiras. Rio de Janeiro - RJ. Brasil. E-mail: terezafelippe@gmail.com.
} 


\section{INTRODUÇÃO}

As doenças cardiovasculares (DCV) são consideradas doenças crônico-degenerativas, nas quais se incluem as neoplasias, doenças respiratórias crônicas e diabetes mellitus ${ }^{1}$. Tendo como característica a etiologia múltipla, associada a deficiências e incapacidades funcionais, que são potencializadas por fatores socioeconômicos, culturais e ambientais, ${ }^{2}$ estes são determinantes na limitação da qualidade de vida da população e na magnitude da morbimortalidade destas doenças.

No Brasil, as DCV representaram a terceira causa de hospitalizações pelo Sistema Único de Saúde (SUS) em 2007, com 1.156.136 internações, sendo a insuficiência cardíaca a causa mais frequente..$^{1-3} \mathrm{~A}$ perspectiva é de aumento destes números entre 40 e $50 \%$, com o aumento da população idosa e melhoria nas condições do tratamento.

Assim, torna-se importante compreender as respostas do indivíduo ao acometimento/doença cardiovascular, considerando a necessidade emergente de autonomia e garantia do exercício profissional da enfermagem baseada em evidências, associando os sintomas e sinais clínicos comumente conhecidos na prática clínica cardiológica em fenômenos, ações e resultados de enfermagem.

Considera-se o diagnóstico de enfermagem como uma linguagem própria e padronizada, conceituada como 0 julgamento clínico das respostas do indivíduo, da família ou da comunidade aos processos vitais ou aos problemas de saúde, que fornecem a base para a seleção das intervenções de enfermagem e para atingir resultados, pelos quais 0 enfermeiro seria o único responsável. ${ }^{4}$

Associar os fatores predisponentes e evidentes a um diagnóstico de enfermagem torna-se útil para determinar clareza e assertividade na conduta de cuidados pela enfermeira e equipe, bem como auxilia a enfermeira na comunicação aos demais membros de equipe sobre a sua atuação multi e interdisciplinar. Além disso, a previsão dos diagnósticos de enfermagem contribui para a construção de futuros protocolos de cuidados à população em estudos e comparativos entre as mesmas populações em outros estudos e cenários. ${ }^{4}$

No Brasil têm sido realizadas pesquisas de identificação de diagnósticos em cardiologia, ${ }^{5-11}$ e sua continuidade permitirá acumular resultados que, uma vez integrados, poderão apoiar decisões sobre os focos clínicos. Diante disso, têm-se como objetivos deste estudo:

- Caracterizar os pacientes hospitalizados com doenças cardiovasculares quanto às características sociodemográficas e clínicas;

- Relacionar os diagnósticos de enfermagem e características definidoras com estas variáveis.

\section{METODOLOGIA}

Trata-se de um estudo de abordagem quantitativa, do tipo descritivo transversal. Teve-se como cenário as enfermarias de cardiologia da clínica médica masculina e feminina, em um hospital universitário de grande porte situado no município de Niterói, Rio de Janeiro, Brasil.

Considerando que temos, neste cenário, 40 pacientes hospitalizados em cada enfermaria separada por sexo, ou seja, 20 homens e 20 mulheres, e estas atendem diversas especialidades, sendo 6 leitos destinados a cardiologia, tratase de uma amostra pequena ao considerarmos a necessidade de estimativa de cálculos do tamanho amostral, para populações finitas. Seria basicamente necessário investigarmos $100 \%$ da amostra, e por isso optamos por 15 pacientes de cada sexo para considerarmos as possíveis perdas e abandono do estudo. Para isso, foi necessário um período de estudo prolongado e exaustivo, entre outubro de 2008 e julho de 2009, considerando que a coleta de dados se deu em até três momentos, pois precisávamos considerar as condições clínicas do paciente e as rotinas hospitalares.

Assim, para seleção dos sujeitos, optou-se por uma amostragem não probabilística com os seguintes critérios de inclusão: ter idade superior a 18 anos e estar hospitalizado há mais de 3 dias; estratificado por sexo, ou seja, iniciamos com um homem, depois uma mulher, e assim sucessivamente, até completarmos 15 homens e 15 mulheres escolhidos por conveniência, que concordassem em participar do estudo, com condições clínicas de responder ao formulário, e que conseguissem o preenchimento completo do instrumento de coleta de dados. Foram considerados como critérios de exclusão a instabilidade clínica que não permitisse condições de responder o formulário, o preenchimento incompleto do instrumento de coleta de dados, a não aceitação em participar do estudo e ter o diagnóstico médico de afecções vasculares não definido no prontuário.

Para realização de tal investigação, foi necessária a construção e validação do instrumento de coleta de dados. Este foi baseado na Taxonomia II da North American Nursing Diagnosis Association - International (NANDA-I) versão 2009/ $2011 .{ }^{4}$ Após ajustes e submissão à apreciação de quatro enfermeiros peritos em cardiologia, através de um teste piloto com cinco pacientes do referido cenário, houve $100 \%$ de concordância sobre a sua sensibilidade e especificidade para levantamento dos diagnósticos de enfermagem em cardiologia, considerando-o assim adequado para coleta de dados.

Durante a coleta de dados, as seguintes técnicas de pesquisa foram utilizadas: formulário e levantamento documental no prontuário. Depois de preenchidos, os 30 instrumentos de coleta de dados foram encaminhados aos enfermeiros peritos, que dispuseram de 1 mês para identificar individualmente os diagnósticos e as características definidoras de tal diagnósticos, ou seja, indícios clínicos que evidenciam a 
presença daquele diagnóstico com certo grau de certeza. ${ }^{4}$ Para que os diagnósticos de enfermagem fossem aceitos para análise, foram considerados aqueles identificados por pelo menos três enfermeiros (concordância de 50\%).

A seleção dos enfermeiros peritos deu-se a partir de critérios sugeridos por Fehring, ou seja, profissionais que tivessem prática assistencial de mais de 5 anos na área de cardiologia, experiência na área de diagnósticos de enfermagem e título de mestre. ${ }^{12}$

A partir dos instrumentos preenchidos, elaboramos um banco de dados no Microsoft Excel 2007, e, selecionando as variáveis do estudo, importamos tal banco para o programa SPSS versão 13.0 para a realização das análises estatísticas.

As análises foram sintetizadas em tabelas para demonstração das principais variáveis sociodemográficas (idade, escolaridade, estado profissional) e clínicas (tempo de internação, tempo de doença, índice de massa corporal, história de doença pregressa, história familiar, causa de internação, hábitos de vida e diagnósticos de enfermagem) relevantes. Assim, na análise descritiva, consideraram-se as variáveis numéricas (idade, tempo de internação, tempo de doença, índice de massa corporal - IMC) através dos cálculos mínimo, máximo, média, de desvio-padrão, de coeficiente de variação (CV) e de percentis. Estas foram submetidas ao teste de KolmogorovSmirnov, a fim de testar a distribuição normal das variáveis na população. Já para as variáveis nominais (escolaridade, estado profissional, história de doença pregressa, história familiar, causa de internação, hábitos de vida e diagnósticos de enfermagem), realizamos cálculo de frequência simples e percentual.

Para associação das características antes analisadas descritivamente à influência na presença dos diagnósticos de enfermagem nesta população, relacionamos as variáveis sociodemográficas e clínicas utilizando o teste Qui-Quadrado ou o teste Exato de Fisher quando necessário - ou seja, quando se tratava de variáveis nominais. Verificada a associação significativa, o risco foi avaliado pelo cálculo da Razão de Chances (Odds Ratio-OR), a um intervalo de confiança de $95 \%$.

Vale considerar o tamanho reduzido da amostra, tendo a razão de chances uma perspectiva de indicativo de associação, e não uma medida exata de risco. Também se atentou, nos casos de diferenças significativas, ao $p$-valor, associado à análise menor que 0,05 .

Este estudo foi aprovado pelo Comitê de Ética e Pesquisa do HUAP/UFF sob o número 165/2007. Consideraram-se os aspectos éticos de acordo com a resolução 196/96 do Conselho Nacional de Saúde (CNS).

\section{RESULTADOS}

Na Tabela 1 apresentamos os dados das variáveis sociodemográficas e clínicas qualitativas, ou seja, nominais dos sujeitos investigados.

Tabela 1. Descrição estatística das variáveis sociodemográficas e clínicas numéricas dos clientes hospitalizados na enfermaria de cardiologia no setor de clínica médica ( $n=30)$. Niterói, 2009.

\begin{tabular}{lcccc}
\hline \multicolumn{1}{c}{ Estatísticas } & $\begin{array}{c}\text { Dias de } \\
\text { internação }\end{array}$ & $\begin{array}{c}\text { Início da } \\
\text { doença } \\
\text { (dias) }\end{array}$ & Idade & IMC \\
\hline Média & 7,63 & 829,30 & 53,93 & 25,9 \\
Desvio-padrão & 8,52 & 1492,02 & 13,11 & 4,7 \\
Mínimo & 1,00 & 1,00 & 21,00 & 17,6 \\
Máximo & 45,00 & 7300,00 & 79,00 & 37,7 \\
Percentil 25 & 2,00 & 30,00 & 48,00 & 23,7 \\
Percentil 50 & 5,50 & 365,00 & 54,00 & 25,1 \\
Percentil 75 & 11,25 & 821,25 & 63,00 & 28,1 \\
Coeficiente de variação & 1,12 & 1,80 & 0,24 & 0,18 \\
p-valor do teste & 0,115 & 0,354 & 0,733 & 0,594 \\
Kolmogorov Sminorv & & & & \\
\hline
\end{tabular}

Obtivemos oito tipos diferentes de causa de internação; $16(53,4 \%)$ dessas internações foram por complicações ocasionadas pela Insuficiência Cardíaca, ou seja, em mais da metade da população. Dada a resposta de incapacidade, dependência, dor e desconfor to ocasionada por esta patologia nos sujeitos, esta será a grande responsável pela associação de tais diagnósticos de enfermagem, como veremos adiante.

Como história pregressa destaca-se o aspecto multifatorial de causas associadas a hospitalizações dos pacientes cardiológicos; estes apresentaram ao todo 75 tipos de doenças associadas, isto é, 2,5 em média por indivíduo, apontando a complexidade dos cuidados de saúde requeridos por tal população.

Dessas doenças, a hipertensão arterial apresentou-se com maior frequência, 26 (86,7\%), seguida da dislipidemia, com 20 (66,7\%). Estas, associadas à hipertensão, como observado nos dados, podem causar complicações como acidente vascular cerebral e/ou infarto. Tivemos também 10 $(33,3 \%)$ sujeitos hospitalizados por infarto agudo do miocárdio; ou seja, houve internações por condições agudas associadas geralmente a outro fator de risco e internações associadas diretamente ao fator estresse, o que ocorreu em metade deles, 5 (13\%). Já o diabetes mellitus e a depressão 
estiveram presentes em 7 (23,3\%) pessoas, estas associadas aos casos de complicações clínicas, prolongamento da internação e falta na adesão terapêutica.

Sobre o histórico familiar de saúde dos sujeitos investigados, houve 48 ocorrências, ou seja, 1,6 em média por sujeito, sendo as mais citadas a hipertensão, o infarto e o diabetes 12 (40\%), evidenciando a importância destas patologias no cenário de promoção e prevenção das doenças cardiovasculares e a consequente demanda por cuidados de enfermagem.

No quesito escolaridade, destacam-se os baixos níveis representados pelo ensino fundamental incompleto na maioria dos pacientes (66,7\%), necessitando aprofundar a associação da influência desta às barreiras socioeconômicas, ao acesso à informação e à adesão terapêutica.

Mais da metade da população, 19 (63,3\%), encontravase profissionalmente inativa um fato preocupante se considerarmos uma média de idade de 53,93 anos, demonstrando uma possível relação entre incapacidade ao trabalho e as DCV e, como veremos adiante, associação a características definidoras de alguns diagnósticos de enfermagem. Mesmo entre os considerados ativos, 11 (36,7\%), ocorreram queixas de afastamento do trabalho e processos indeferidos de aposentadoria por invalidez.

Como fator de risco, houve $25(83,3 \%)$ sedentários, $20(66,7 \%)$ tabagistas e $19(63,3 \%)$ etilistas, totalizando 64 fatores - 2,1 em média por indivíduo; estes, além de contribuir para o desenvolvimento das DCV, dificultam o seu controle, sendo importante a conscientização e educação do paciente para adoção de hábitos saudáveis de vida.

Na Tabela 2 observa-se o resumo estatístico das variáveis numéricas discretas e contínuas relacionadas às características do sujeito, como idade e IMC, bem como da hospitalização e tempo decorrido desde o início da doença, em dias.

Tabela2. Descrição das variáveis sociodemográficas e clínicas nominais dos clientes hospitalizados na enfermaria de cardiologia no setor de clínica médica ( $n=30)$. Niterói, 2009.

\begin{tabular}{|c|c|c|}
\hline Variáveis & $\mathbf{n}$ & $(\%)$ \\
\hline \multicolumn{3}{|l|}{ Causa de internação } \\
\hline Insuficiência cardíaca & 16 & 53,4 \\
\hline Infarto agudo do miocárdio & 05 & 16,7 \\
\hline Angina instável & 03 & 10,0 \\
\hline Fibrilação atrial & 02 & 6,7 \\
\hline Bloqueio atrioventricular & 01 & 3,3 \\
\hline $\begin{array}{l}\text { Correção de níveis tensionais de } \\
\text { pressão }\end{array}$ & 01 & 3,3 \\
\hline Realização de cateterismo & 01 & 3,3 \\
\hline $\begin{array}{l}\text { Troca de gerador de marca- } \\
\text { passo }\end{array}$ & 01 & 3,3 \\
\hline \multicolumn{3}{|l|}{ História pregressa } \\
\hline Hipertensão arterial & 26 & 86,7 \\
\hline Dislipidemia & 20 & 66,7 \\
\hline Infarto agudo do miocárdio & 10 & 33,3 \\
\hline Diabetes mellitus & 07 & 23,3 \\
\hline Depressão & 07 & 23,3 \\
\hline Estresse & 05 & 13,0 \\
\hline \multicolumn{3}{|l|}{ História familiar } \\
\hline Hipertensão arterial & 12 & 40,0 \\
\hline Infarto agudo do miocárdio & 12 & 40,0 \\
\hline Diabetes mellitus & 12 & 40,0 \\
\hline Acidente vascular encefálico & 07 & 23,3 \\
\hline Insuficiência cardíaca & 05 & 16,7 \\
\hline \multicolumn{3}{|l|}{ Escolaridade } \\
\hline Analfabeto & 02 & 6,7 \\
\hline Fundamental incompleto & 20 & 66,7 \\
\hline Fundamental completo & 02 & 6,7 \\
\hline Médio incompleto & 01 & 3,2 \\
\hline Médio completo & 01 & 6,7 \\
\hline Superior & 01 & 6,7 \\
\hline \multicolumn{3}{|l|}{ Estado profissional } \\
\hline Ativo & 11 & 36,7 \\
\hline Inativo & 19 & 63,3 \\
\hline \multicolumn{3}{|l|}{ Hábitos de vida } \\
\hline Sedentarismo & 25 & 83,3 \\
\hline Tabagismo & 20 & 66,7 \\
\hline Etilismo & 19 & 63,3 \\
\hline
\end{tabular}


Obtivemos como média de idade em anos 53,93 ( $\pm 13,11)$, esta, com distribuição normal na amostra ( $p$-valor $=0,733$ ) ao teste de KolmogorovSminorv (teste KS), variando entre21 (outline) a 79 anos; portanto, salvo alguns casos, principalmente os agudos, trata-se de uma população adulta eidosa.

Dos 30 pacientes avaliados, $50 \%$ tinham IMCacima de 25,1, ou seja, estavam em situação de sobrepeso; $25 \%$ dos dientes apresentavam IMC acima de 28,1, caracterizando situação de obesidade. 0 IMC mínimo encontradofoi 17,6eomáximo 37,6,tendo comovalor médio de 25,9 44,7, resultando em um coeficiente de variação de 0,18 . Pelo teste de Kolmogorov Smirnov, a distribuição do IMC dos clientes hospitalizados apresentou distribuição normal ( $p$-valor $=0,594)$.

A taxa de média de permanência durante a hospitalização permaneceu em 7,63 dias $( \pm 8,52)$ sendo a mediana de 5,5 dias. Dado o coeficiente de variação de 1,12, considera-se a variável consistentemente heterogênea, com tempo mínimo de 1 dia e máximo de 45 dias, tendo em $25 \%$ dos casos o tempo de internação ultrapassado em 11 dias, o que indica tratar de casos complexos com alta taxa de permanência e custos hospitalares. 0 teste KS mostrou que não há evidências para rejeitar a hipótese de que esta variável tem distribuição normal na população ( $p$-valor=0,115).

0 tempo desde o início da doença, em dias, também apresentou alta variabilidade entre os pacientes, visto que 0 coeficiente de variação foi de 1,80 seguindo distribuição normal ( $p$-valor=0,354, teste KS). Teve-se como valor mínimo 30 dias e máximo de 7.300 dias (20 anos), e a mediana demonstra que pelo menos $50 \%$ dos pacientes apresentavam doença a pelo menos 365 dias (1 ano). Esta variabilidade pode estar relacionada com a cronicidade de algumas doenças, que acometem os pacientes muitas vezes de maneira progressiva, iniciando com a hipertensão arterial e levando, após longo período, a insuficiência cardíaca, via final da maioria delas.

Foram documentados 198 diagnósticos de enfermagem, com média de 6,6 por paciente $( \pm 2,64)$ e 35 títulos diagnósticos distintos, estes demonstrados na Tabela 3 quanto à sua distribuição por domínios.

Tabela 3. Distribuição dos diagnósticos de enfermagem segundo domínios nos pacientes da enfermaria de cardiologia no setor de clínica médica ( $n=30)$. Niterói, 2009.

\begin{tabular}{|c|c|c|c|}
\hline Domínios & Diagnósticos de enf ermagem & $\mathbf{N}$ & $\mathrm{N}(\%)$ \\
\hline \multirow[t]{2}{*}{ Promoção da saúde } & Manutenção ineficaz da saúde & 08 & 26,7 \\
\hline & Autocontrole ineficaz da saúde & 03 & 10 \\
\hline \multirow[t]{3}{*}{ Nutrição } & $\begin{array}{l}\text { Nutrição desequilibrada: menos do que } \\
\text { as necessidades corporais }\end{array}$ & 05 & 16,7 \\
\hline & Deglutição prejudicada & 02 & 6,7 \\
\hline & $\begin{array}{l}\text { Nutrição desequilibrada: mais do que as } \\
\text { necessidades corporais }\end{array}$ & 01 & 3,3 \\
\hline \multirow[t]{4}{*}{ Eliminação/troca } & Eliminação urinária prejudicada & 11 & 36,7 \\
\hline & Constipação & 06 & 20 \\
\hline & Diarreia & 01 & 3,3 \\
\hline & Insônia & 14 & 46,7 \\
\hline \multirow[t]{7}{*}{ Atividade/repo uso } & Deambulação prejudicada & 01 & 3,3 \\
\hline & Débito cardíaco diminuído & 17 & 56,7 \\
\hline & Intolerância à atividade & 11 & 36,7 \\
\hline & Perfusão tissular ineficaz & 09 & 30 \\
\hline & $\begin{array}{l}\text { Déficit no autocuidado para } \\
\text { banho/higiene }\end{array}$ & 04 & 13,3 \\
\hline & Déficit no autocuidado para vestir-se & 02 & 6,7 \\
\hline & Fadiga & 01 & 3,3 \\
\hline \multirow[t]{4}{*}{ Percepção/cognição } & Percepção sensorial perturbada (visual) & 16 & 53,3 \\
\hline & $\begin{array}{l}\text { Percepção } \\
\text { (auditiva) }\end{array}$ & 02 & 6,7 \\
\hline & $\begin{array}{l}\text { Conhecimento deficiente relacionado à } \\
\text { doença/tratamento }\end{array}$ & 02 & 6,7 \\
\hline & Memória prejudicada & 02 & 6,7 \\
\hline \multirow[t]{4}{*}{ Autopercepção } & Sentimen to de impotência & 03 & 10 \\
\hline & Baixa autoestima situacional & 02 & 6,7 \\
\hline & Baixa autoestima crônica & 01 & 3,3 \\
\hline & Distúrbio da imagem corporal & 04 & 13,3 \\
\hline Relacionament os & Amamentação interrompida & 01 & 3,3 \\
\hline Sexualidade & Disfunção sexual & 11 & 36,7 \\
\hline \multirow{2}{*}{$\begin{array}{l}\text { Enfrentamento/tolerância ao } \\
\text { estresse }\end{array}$} & Ansiedade & 23 & 76,7 \\
\hline & Tristeza crônica & 02 & 6,7 \\
\hline \multirow[t]{4}{*}{ Segurança/proteção } & Risco de infecção & 01 & 3,3 \\
\hline & Dentição prejudicada & 06 & 20 \\
\hline & Risco de quedas & 05 & 16,7 \\
\hline & Hipertermia & 01 & 3,3 \\
\hline \multirow[t]{3}{*}{ Conf orto } & Dor aguda & 21 & 70 \\
\hline & Dor crônica & 03 & 10 \\
\hline & Náusea & 06 & 20 \\
\hline
\end{tabular}


Os diagnósticos de enfermagem com frequência acima de 50\% foram: ansiedade 23 (76,7\%), dor aguda 21 (70,7\%), débito cardíaco diminuído 17 (56,7\%) e percepção sensorial perturbada (visual) 16 (56,3\%). Já os diagnósticos com frequência menor que $50 \%$ e maior ou igual a $30 \%$ compreenderam: insônia 14 (46,7\%), disfunção sexual e intolerância à atividade 11 (36,7\%) e perfusão tissular ineficaz $9(30,0 \%)$. Os outros 27 diagnósticos tiveram frequência inferior a $30 \%$, variando entre $8(26,7 \%)$ e $1(3,3 \%)$.

Identificou-se o diagnóstico de ansiedade em 12 homens e 11 mulheres, sendo as características definidoras mais típicas, com frequência acima de 50\%: agitação, $(95,7 \%)$, seguida do estado ansioso $(69,6 \%)$, insônia $(65,2 \%)$ e preocupações expressas em razão de mudanças e eventos da vida (52,2\%). Já a dor aguda foi indicada no formulário por 9 homens e 12 mulheres; contudo, o relato verbal de dor foi apresentado em $100 \%$ dos clientes.

Destaca-se o débito cardíaco diminuído, identificado em quase metade da população em igual distribuição entre os sexos, 9 homens e 8 mulheres. Este foi observado com maior frequência em pacientes com insuficiência cardíaca, com $p$-valor de 0,003 ao teste qui-quadrado, o que foi corroborado pelo teste de razão de chances ao demonstrar que o paciente com insuficiência cardíaca apresenta 13,20 vezes maior chance de ter o diagnóstico de enfermagem do que um paciente sem esta doença (IC para a OR, $95 \%=2,11-82,50$ ).

As características definidoras mais presentes foram: dispneia $(70,6 \%)$, distensão de veia jugular e edema $(64,7 \%) \mathrm{e}$ fadiga $(58,8 \%)$. Cabe ressaltar que estes tiveram média de IMC significativamente mais baixo $(24,3)$ do que os outros sujeitos do estudo (28,3), com $p=0,029$ ao teste t-de Student.

0 diagnóstico de enfermagem percepção sensorial perturbada (visual), presente mais nos homens (12) que nas mulheres (4), teve como características definidoras presentes em 15 pacientes $(93,8 \%)$ mudança na acuidade visual, e distorções sensoriais, em 13 (81,3\%).

Ainsônia apresentou distribuição equiparável entre os sexos, com 08 homens e 06 mulheres, e associação significativa com 0 infarto agudo do miocárdio (IAM) ( $p$-valor $=0,014$, teste quiquadrado). Os pacientes que foram internados por IAM tiveram 1,55 vez mais chance de apresentarem insônia (IC 95\%: $(1,05-2,29)$ do que os que não tinham IAM. A característica definidora mais comum foi o relato da dificuldade para permanecer dormindo $(76,5 \%)$.

Quanto à intolerância à atividade diagnosticada em 08 homens e 03 mulheres, apesar da aparente diferença de frequência entre os sexos, não apresentou associação estatística significativa ( $p$-valor $=0,058$, teste qui-quadrado). Tal diagnóstico predominou nos pacientes com insuficiência cardíaca ( $p$-valor $=$ 0,003 , teste qui-quadrado), ou seja, houve 12,60 mais chance de serem diagnosticados com intolerância à atividade (IC 95\% $=1,99-79,43)$. A característica definidora identificada em $100 \%$ dos clientes foi a dispneia, seguida de alterações eletrocardiográficas refletindo arritmias em 5 pacientes $(72,7 \%)$ e desconforto aos esforços em $63,6 \%$ dos sujeitos.

0 diagnóstico de enfermagem disfunção sexual foi encontrado em 7 mulheres e 4 homens, e esteve associada à idade superior a 60 anos ( $p=0,041$ do teste qui-quadrado). Apresenta como características definidoras com frequência acima de 50\%: verbalização do problema (de disfunção sexual), em 9 $(81,8 \%)$, seguida de limitações reais impostas pela doença, em $7(63,6 \%)$ e déficit percebido de desejo sexual, em $6(54,5 \%)$.

A eliminação urinária prejudicada foi mais presente entre os homens (9) e somente em 2 mulheres, ou seja, 9,75 vezes mais chances em homens (IC 95\% = 1,59-59,69) do que em mulheres. Também esteve associada à insuficiência cardíaca ( $p=$ 0,029), e, com base no teste de razão de chances, os pacientes com esta doença possuíam 5,77 vezes mais chances de serem identificados com este diagnóstico de enfermagem (IC 95\% = $1,11-29,84)$. A característica definidora com mais de $50 \%$ de evidência foi a nictúria.

Os outros diagnósticos de enfermagem identificados com frequência inferior a $30 \%$ foram: manutenção ineficaz da saúde; autocontrole ineficaz da saúde; nutrição desequilibrada: menos do que as necessidades corporais; nutrição desequilibrada: mais do que as necessidades corporais; deglutição prejudicada; constipação; diarreia; deambulação prejudicada; perfusão tissular ineficaz; déficit do autocuidado para banho/higiene; déficit do autocuidado para vestir-se; percepção sensorial perturbada (auditiva); conhecimento deficiente relacionado à doença/ tratamento; memória prejudicada; sentimento de impotência; baixa autoestima situacional; baixa autoestima crônica; distúrbio da imagem corporal; amamentação interrompida; tristeza crônica; risco de infecção; dentição prejudicada; risco de quedas; hipertermia; dor crônica; e náusea.

\section{DISCUSSÃO}

Foram documentados 198 diagnósticos de enfermagem na população hospitalizada por distúrbios cardiovasculares. Tal expressividade pode ser considerada alta, visto que cada diagnóstico de enfermagem a ser resolvido exige do enfermeiro o planejamento de intervenções e avaliação constante para a mensuração dos resultados. Pela identificação dos diagnósticos, evidenciamos a gama de cuidados requeridos por tal população e o subsequente apoio à implementação nas demais fases do processo de enfermagem.

Ressalta-se a média elevada da taxa de hospitalização, indicando uma complexidade de respostas à doença e ao tratamento que exigem atenção dos profissionais de enfermagem na avaliação e no raciocínio diagnóstico, evitando, assim, possíveis falhas de julgamento clínico; daí a importância de tal investigação. ${ }^{1-4}$

Pode-se considerar baixa a média de idade no início dos sintomas entre os países em desenvolvimento, e isso está associado à dificuldade de acesso ao sistema de saúde e às barreiras econômicas. Nos países em desenvolvimento, os indivíduos são provavelmente acometidos mais precocemente 
pelas doenças cardiovasculares e apresentam menor adesão terapêutica, acarretando em mortes mais precoces. ${ }^{1-4}$

Os dados ainda demonstraram uma tendência dos pacientes ao sobrepeso, considerada como um fator de risco para a insuficiência cardíaca, que é a causa mais frequente de internação entre 2000 e 2007, com hospitalizações ocorrendo em indivíduos acima de 60 anos em uma proporção de dois terços da população, e a taxa de mortalidade variando de acordo com a faixa etária, sendo maior nos idosos e nos menores de 20 anos. ${ }^{1-2}$

Por se tratar de uma síndrome que traz limitações à qualidade de vida ao indivíduo, principalmente devido à tríade fadiga-dispneiaedema, pacientes com insuficiência cardíaca também sofrem com a aposentadoria precoce e com o absenteísmo, o que causa um custo indireto para o país, refletindo a necessidade de implementação de política de facilitação de acesso aos medicamentos de uso crônico de âmbito ambulatorial. ${ }^{1-3}$

Em relação à história familiar, considera-se o infarto agudo do miocárdio um indicador de risco intermediário de morte súbita ou acidente vascular encefálico em familiares de primeiro grau ocorrido antes dos 50 anos,e, ainda, como fator de risco cardiovascular.Considera-se a presença de doença arteriocoronariana prematura (familiar de primeiro grau do sexo masculino com menos de 55 anos e do sexo feminino com menos de 65 anos) $\cdot^{1-4}$

O estudo EPICA-Niterói (Epidemiologia da Insuficiência Cardíaca em Niterói) avaliou e comparou o perfil demográfico e socioeconômico, bem como características clínicas de pacientes admitidos em hospitais públicos e privados por IC descompensada. Foi notável a diferença socioeconômica dos pacientes hospitalizados em setor público, onde $56 \%$ eram analfabetos e $57 \%$ possuíam renda de até 1 salário mínimo. ${ }^{3}$

Alguns estudos demonstraram que o acometimento por doenças cardiovasculares ocasiona sinais e sintomas emocionais associados ao medo da morte e da dor. ${ }^{15-17} \mathrm{Em}$ um estudo sobre diagnósticos de enfermagem com 80 pacientes com infarto agudo do miocárdio, a ansiedade esteve presente em $93,0 \%$ dos pacientes. Eem outro estudo com pacientes em pré-operatório de cirurgia cardíaca, $47,1 \%$, no transoperatório, 68,2\% também apresentaram ansiedade. ${ }^{8,11}$

A ansiedade combinada com a depressão podem predizer eventos cardiovasculares. ${ }^{16-17}$ Em um estudo realizado com 490 mulheres com suspeita de isquemia miocárdica acompanhadas por 6 anos, 75 mulheres apresentaram eventos cardiovasculares e 18 foram a óbito decorrente damesma causa. Entreas mulheres com suspeita de isquemia miocárdica, o valor de sintomas de depressão para predizer eventos cardiovasculares variou em função da gravidade da ansiedade comórbida. Estes resultados sugerem que a utilidade clínica das medidas de depressão pode ser melhorada, utilizando-as em combinação com medidas de ansiedade. ${ }^{15-17}$

Outro estudo teve o objetivo de relatar as experiências de uma amostra de 24 sujeitos hospitalizados com doença isquêmica do coração pela primeira vez entre a fase de internação até a alta. 0 principal resultado foram sinais e sintomas de confusão e ansiedade acerca do autocuidado e progressão da doença, principalmente devido ao medo de morrer. Estes achados sugerem a importância das intervenções de enfermagem para a ansiedade do paciente e redução de confusão através da gestão dos sintomas de doença cardíaca durante a hospitalização e acompanhamento no pós-alta em âmbito ambulatorial. ${ }^{16}$

Estudos têm comprovado a presença do diagnóstico de enfermagem dor em pacientes hospitalizados. ${ }^{11,14}$ Tais estudos corroboram o resultado apresentado, visto que a dor é um diagnóstico importante a ser evidenciado na prática clínica, principalmente em sua complexidade biopsicossocial. ${ }^{5}$

Sobre débito cardíaco diminuído, obtivemos resultados aproximados aos achados de estudos internacionais de registros de pacientes hospitalizados com insuficiência cardíaca, o segundo diagnóstico mais identificado. ${ }^{11}$

A característica definidora dispneia, na insuficiência cardíaca, pode ocorrer por atividade mínima ou moderada, ou também em repouso, devido à incapacidade do coração em manter um débito cardíaco suficiente para satisfazer as necessidades teciduais de oxigênio, bem como da redução do fluxo sanguíneo periférico, daí também as associações com a intolerância à atividade. ${ }^{18}$

A turgência ou distensão de veia jugular ocorreu devido ao aumento na pressão venosa, consequente da fração de ejeção diminuída pela redução no volume ventricular ejetado. A disfunção sistólica surgiu quando houve fração de ejeção menor que 35$40 \%$. Já a disfunção diastólica ocorre quando, além da fração de ejeção de $45 \%$ a $55 \%$ há presença de hipertrofia do ventrículo esquerdo e aumento de átrio esquerdo. A média de fração de ejeção encontrada nos pacientes com insuficiência cardíaca foi de aproximadamente $31 \%$ neste estudo. Isso mostra que a característica definidora aqui discutida teve abrangência em pacientes com função cardíaca prejudicada, mostrando a importância deste achado clínico junto a outros dados da anamnese e exame físico, na sustentação do diagnóstico de débito cardíaco diminuído. $^{18}$

0 edema foi outro achado clínico associado à presença do débito cardíaco, evidenciado pela infiltração no espaço intersticial, mais comumente encontrado neste estudo em membros inferiores, em uma escala aproximada de $+3 /+4 a+4 /+4 .{ }^{18}$

Finalmente, a fadiga, também característica definidora dos pacientes com débito cardíaco, definida como fraqueza, é decorrente da hipoperfusão aos músculos. Os pacientes referiram sensação de "peso" nos braços e pernas. ${ }^{18}$ Este resultado pode ser explicado pelo fato de estes terem seguido um esquema terapêutico farmacológico, restrição hídrica e dieta específica para insuficiência cardíaca durante a hospitalização.

Sobre percepção visual perturbada (visual) tem-se que, à medida que novas células se formam na superfície externa do cristalino do olho, as antigas células centrais se acumulam e ficam amareladas, rígidas, densas e turvas. 0 cristalino torna-se menos flexível; e o ponto máximo do foco, mais distante. Essa condição, a presbiopia, exige o uso de óculos de leitura para ampliar os objetos e, geralmente, começa aos 50 anos, média de idade encontrada na população em estudo. ${ }^{18}$

Padrão alterado de sono pode ser uma resposta humana comum à doença e à hospitalização, mas merece atenção especial 
em pacientes com doença cardiovascular, pois podem ocorrer sono ineficaz e alterações fisiológicas adversas. A função cardíaca prejudicada por qualquer causa pode gerar sintomas como precordialgia e dispneia, que interferem no sono. ${ }^{18}$

0 diagnóstico de enfermagem intolerância à atividade esteve presente em $100 \%$ dos pacientes hospitalizados ( $n=26$ ) com insuficiência cardíaca em um estudo nacional. ${ }^{9} \mathrm{~A}$ intolerância à atividade nesta doença pode ser justificada pelo desconfor to respiratório, fadiga e palpitação que estes clientes sentem ao desenvolver suas atividades diárias, devido à incapacidade do coração em manter um débito cardíaco suficiente para satisfazer as necessidades teciduais de oxigênio e da redução do fluxo sanguíneo periférico. ${ }^{18}$

Muitos pacientes não retornam à atividade sexual após um evento cardíaco devido a diversos fatores, como as mudanças fisiológicas decorrentes do processo de envelhecimento humano, as disfunções induzidas por medicamentos e a presença de comorbidades que causam alterações vasculares, além do impacto emocional da doença cardíaca. ${ }^{16-18}$ Isso corrobora os achados deste trabalho, que demonstram diferença na idade dos grupos com e sem disfunção sexual, sendo atingidos significativamente os pacientes mais velhos. ${ }^{16}$

Sobre a eliminação urinária em pacientes com insuficiência cardíaca seria comum o sintoma de nictúria, pelo uso contínuo de diuréticos no tratamento. ${ }^{2,18}$ Estes são utilizados em associação aos inibidores da enzima conversora de angiotensina (ECA) ou digitálicos, aumentando a natriurese e a diurese, reduzindo o volume intravascular, a pré-carga, a congestão visceral e, consequentemente, os sintomas da doença. Pacientes nesta condição tendem a urinar mais a fim de reduzir a congestão do organismo e edema. ${ }^{1-2}$

É importante que o enfermeiro esteja atento na identificação das respostas do paciente hospitalizado à doença e tratamento, pois estes determinam mais do que a tríade de sintomas edema-fadiga-dispneia, tão discutida e enfatizada na literatura e evidenciada neste estudo pelos principais diagnósticos encontrados: ansiedade; dor aguda; débito cardíaco diminuído; percepção sensorial perturbada (visual); insônia; disfunção sexual e eliminação urinária prejudicada.

\section{CONCLUSÃO}

0 tempo de internação médio dos pacientes com doenças cardiovasculares hospitalizados foi de 7 dias, com cerca de 2 anos do início do aparecimento da doença até o período de internação, sendo a média de idade de 53,9 anos. A maioria deles possui o ensino fundamental incompleto, é inativa, tem como principal condição pregressa a hipertensão arterial, está em situação de sobrepeso e tem como principal causa de internação atual a insuficiência cardíaca. A história familiar identificada foi de hipertensão arterial, diabetes mellitus e infar to agudo do miocárdio. Cabe destacar que esta amostra apresentou uniformidade em suas características.
Na análise dos diagnósticos de enfermagem, os de maior frequência foram: ansiedade, dor aguda, débito cardíaco diminuído, percepção sensorial perturbada (visual), insônia, intolerância à atividade, disfunção sexual e eliminação urinária prejudicada. Foi possível, portanto, conhecer o cliente com doença cardíaca em condição de hospitalização e analisar relações entre suas características.

A ansiedade apareceu como o diagnóstico mais encontrado, com ausência de relação significativa com as variáveis de caracterização investigadas nestes clientes. 0 diagnóstico de enfermagem dor aguda foi estatisticamente relacionado com outro diagnóstico, o de débito cardíaco diminuído. Pela razão de chances, um cliente com insuficiência cardíaca apresenta maior chance de ser diagnosticado com débito cardíaco diminuído e intolerância à atividade do que aqueles que não têm tal doença.

Sobre percepção visual perturbada (visual), tivemos uma chance aumentada de os clientes do sexo masculino apresentarem este diagnóstico do que as do sexo feminino. Já a insônia esteve relacionada estatisticamente com a causa de hospitalização, o infarto agudo do miocárdio. A disfunção sexual apareceu de modo significativo, ao teste qui-quadrado, nos clientes com idade acima de 60 anos. E, a ocorrência de eliminação urinária prejudicada se mostrou bem mais provável nos clientes com insuficiência cardíaca.

Portanto, este estudo mostrou-se relevante por sua originalidade, tanto no objeto, quanto no método, em apresentar e validar com peritos a identificação de diagnósticos de enfermagem e suas características definidoras em clientes com distúrbios cardiovasculares hospitalizados nas enfermarias de clínica médica feminina e masculina, relacionando-os com as variáveis sociodemográficas e clínicas evidenciadas.

A descrição dos diagnósticos de enfermagem contribui para a análise das respostas à doença cardiovascular, com foco no objeto de trabalho do enfermeiro que, associado às questões de gênero, culturais, demográficas, sociais, econômicas, bem como às condições do tratamento médico e espirituais, possibilita uma investigação holística, embasada em uma linguagem internacional proposta pela NANDA-I.

Como limitação deste estudo, declara-se o reduzido tamanho amostral; assim, sugere-se a realização de novos estudos com amostras ampliadas e em múltiplos cenários, pois estudos sobre a frequência dos diagnósticos de enfermagem em uma população contribuem para organizar a base de conhecimento de enfermagem, a prever os cuidados necessários a uma população específica e a orientar a organização dos serviços hospitalares e os programas de educação dos clientes e profissionais. Além disso, podem estimar a probabilidade de determinadas populações apresentarem diagnósticos, favorecendo a previsão das necessidades de enfermagem, da carga de trabalho do enfermeiro e estimativas dos resultados que podem ser obtidos com os cuidados de enfermagem. 


\section{REFERÊNCIAS}

1- Ministério da Saúde (BR). Diretrizes e recomendações para o cuidado integral de doenças crônicas não-transmissíveis: promoção da saúde, vigilância, prevenção e assistência.Brasília(DF); 2008.

2-Bocchi EA, Marcondes-Braga FG, Ayub-Ferreira SM, Rohde LE, Oliveira WA, Almeida DR, et al. $3^{\mathrm{a}}$ Diretriz Brasileira de Insuficiência Cardíaca Crônica. Arq Bras Cardiol. 2009 junho; 92 (6 supl.1): 1-71.

3-Tavares LR, Victer H, Linhares JM, Barros CM, Oliveira MV, Pacheco LC, et al. Epidemiologia da insuficiência cardíaca avançada em Niterói- Projeto EPICA Niterói. Arq Bras Cardiol. 2002 fev 82(2): 121-24.

4-North American Nursing Association-NANDA Diagnósticos de enfermagem da nanda: definições e classificação-2009-2011. São Paulo: Artmed; 2010.456 p.

5- Galdeano LE, Rossi LA, Pezzuto TM. Diagnósticos de enfermagem de pacientes no período pré-operatório de cirurgia cardíaca. Rev Esc Enferm USP. 2004 mar/abr; 38(3): 307-16.

6- Galdeano LE, Rossi LA, Nobre LF, Ignácio DS. Nursing diagnosis in the intra-operative period of cardiac surgery. Rev Latino-am. Enfermagem. $2003 \mathrm{mar} / \mathrm{abr} ; 11(2): 199-206$.

7-Rocha LA, Maia TF, Silva LF. Diagnósticos de enfermagem em pacientes submetidos à cirurgia cardíaca. Rev Bras Enferm. 2006 maio/jun; 59(3). 8-Silva RCG, Cruz DALM. Identificação dos diagnósticos de enfermagem, características definidoras e fatores de risco em pacientes valvopatas. Rev Soc Cardiol Est São Paulo.2002 jul;12(2): 1-7.

9- Assis CC, Barros ABL. Nursing diagnoses in patients with congestive heart failure. Intern J Nurs Terminol Classif. 2003 0ct; 14 (supl s4): 1.

10-Barros ALB, Gomes IM. Perfil dos diagnósticos de enfermagem de pacientes internados com infarto agudo do miocárdio. Acta Paul Enferm. 2000; 3(n.esp p II):105-08.

11-Scherb CA, Head BJ, Mass ML, Swanson EA, Moorhead S, Reed D, et al. Most frequent nursing diagnoses, nursing interventions, and nursingsensitive patient outcomes of hospitalized older adults with heart failure: part 1. Intern I Nurs Terminol Classif. 2011 jan/mar; 22(1): 13-22.

12-Fehring R. The Fehring Model. In: Johnson RMC, Paquette M, editors. Classification of nursing niagnoses: proceedings of the $10^{\mathrm{a}}$ Conference of North American Nursing Diagnosis Association. Lippincott; 1994. p. 55-62.

7- Volpato MP, Cruz DALM. Diagnósticos de enfermagem de pacientes internadas em unidade medico-cirúrgica. Acta Paul Enferm. 2007 abr/jun; 20(2):119-24.

13-Fontes CMB, Cruz, DALM. Diagnósticos de enfermagem documentados para pacientes de clínica médica. Rev Esc Enferm USP. 2007 set; 20(3): 395-402.

14- Batista DCS, Cruz DALM, Pimenta, CAM. Publicações sobre dor e diagnóstico de enfermagem em uma base de dados brasileira. Rev Gaucha Enferm. 2008 set; 29(3): 461-67.
16- Rutledge ST, Linke SE, Krantz DS, Johnson D, Bittner V, Eastwood J, et al. Comorbid depression and anxiety symptoms as predictors of cardiovascular events: Results from the NHLBI-Sponsored Women's Ischemia Syndrome Evaluation (WISE). Psychosom Med. 2009 Nov ; 71(9): 958-64.

17-Cavalcanti ACD, Coelho MJ. A linguagem como ferramenta do cuidado do enfermeiro em cirurgia cardíaca. Esc Anna Nery. 2007 jun; 11(2): 220-26.

18-Givertz MM, Colucci WS, Braunwald E. Aspectos clínicos de insuficiência cardíaca. In: Zipes DP, Libby P, Bonow RO. Braunwald E. Braunwald. Tratado de Doenças Cardiovasculares; 2009. p.539-68. 